Supporting Information: "Spectral editing of organic mixtures into pure components using NMR spectroscopy_and ultra-viscous solvents.” for Analytical Chemistry prepared October $12^{\text {th }}, 2007$.

Including this cover page, the document contains two pages.

\title{
Spectral editing of organic mixtures into their pure components using NMR spectroscopy and ultra-viscous solvents
}

\author{
André J. Simpson*, Gwen Woods and Omid Mehrzad \\ Department of Chemistry, University of Toronto at Scarborough, Canada
}

${ }^{*}$ Corresponding author. Tel: 1-416-287-7547; Fax: 1-416-287-7279; E-mail address: andre.simpson@utoronto.ca 


\section{Practical Guide: Tips and Tricks}

1) Working with the viscous $1000 \mathrm{~N}$ Halocarbon oil. Due to the high viscosity of the oil, we recommend measuring the oil on a weight basis. The density of the $1000 \mathrm{~N}$ oil is approximately $1.98 \mathrm{~g} / \mathrm{mL}$ at room temperature. A plastic disposal pipette provides a convenient tool for transferring the oil.

2) Sample Prep. First, dissolve the mixture/compound fully in the pure solvent (for example D-chloroform). Then add the solvent-mix to the oil (pre-weighed amount in a GC vial), sonicate, vortex and transfer to an NMR tube. Do not try to dissolve the compound of mixture directly in the oil/solvent mix.

3) Locking the Spectrometer. For all samples the lock power may need to be adjusted prior to automatic locking. We have found that our spectrometer (Bruker, Avance I) can lock directly on all samples with the exception of 90:10 oil:chloroform. However, for all samples the lock power may/will need to be adjusted prior to automatic locking. For 90:10 oil:chloroform (and possibly other 90:10 mixes) an external lock is required. There are many ways of achieving an external lock; the simplest and most cost effective way is by inserting a small capillary filled with deuterated solvent down the center of the oil sample. Suitable capillaries are available from Wilmad Glass Inc and other NMR tube manufactures.

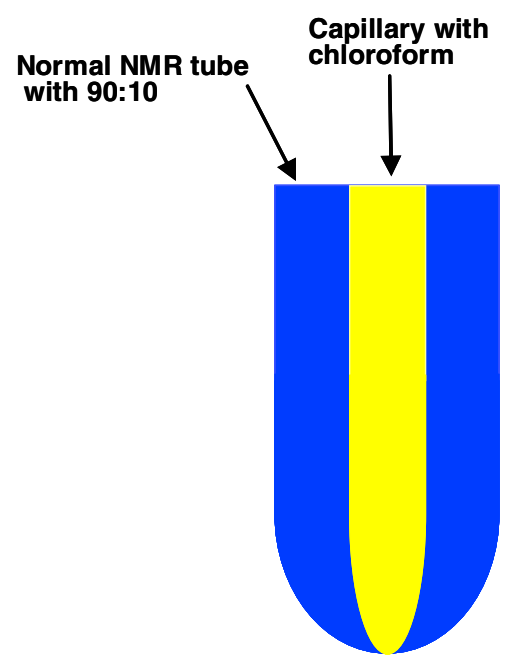

4) Performing an NMR experiment. Perform actions in the following order :

a) Lock and Shim the spectrometer at room temperature.

b) Lower the temperature.

c) Match and Tune the Spectrometer once the temperature has been reached. (Dropping the temperature can shift the "wobb curve" significantly.) 